Ingeniare. Revista chilena de ingeniería, vol. $15 \mathrm{~N}^{\circ} 1,2007, \mathrm{pp} .65-75$

\title{
ESTUDIO COMPARATIVO DE MODELOS NUMÉRICOS PARA EL SEGUIMIENTO DE INTERFACES MÓVILES: ESTUDIO DEL DERRAME DE UNA COLUMNA DE LÍQUIDO
}

\section{COMPARATIVE STUDY OF NUMERICAL MODELS FOR MOVING INTERFACE PROBLEMS: ANALYSIS OF THE COLLAPSE OF A LIQUID COLUMN}

\author{
Juan Pablo Barraza ${ }^{1} \quad$ Marcela Andrea Cruchaga $^{1}$ \\ Recibido 27 de diciembre de 2005, aceptado 23 de agosto de 2006 \\ Received: December 27, 2005 Accepted: August 23, 2006
}

\begin{abstract}
RESUMEN
En este trabajo se presenta la simulación numérica del problema del derrame de una columna de líquido. El análisis numérico se realiza utilizando el método de volúmenes finitos y la técnica conocida como VOF para el seguimiento de la superficie interfaz. Se asume que el problema puede ser descrito por las ecuaciones de cantidad de movimiento y continuidad para un fluido incompresible. Dichas ecuaciones se resuelven tanto para el fluido como para el aire en todo el dominio de análisis en conjunto con la ecuación que describe el movimiento de la interfaz. Se estudia el comportamiento de dos fluidos de diferente viscosidad. Se evalúa la respuesta frente a cambios en la discretización espacial y la incidencia en los resultados cuando se utiliza un modelo turbulento para la descripción del flujo. Los resultados obtenidos para la posición de la superficie libre se comparan con las predicciones calculadas con una formulación de elementos finitos de malla fija y, además, con mediciones experimentales reportadas en la literatura.
\end{abstract}

Palabras clave: Interfaces móviles, superficies libres, problema de dos medios, mecánica de fluido computacional, verificación y validación.

\section{ABSTRACT}

The simulation of the collapse of a liquid column problem is presented in this work. The numerical analysis is done using a finite volume technique, where the well-known VOF technique is applied to describe the free surface motion. The adopted mathematical model is given by the Navier-Stokes equations for incompressible flows. These equations are solved in the whole domain occupied by both fluids (liquid and air) together with the interface motion equation. The study focuses on the analysis of two liquids with different viscosities. The response is assessed by considering various spatial discretizations, when a turbulent model is applied. The computed numerical results are compared with those obtained using a fixed-mesh finite element technique and with experimental measurements reported in the literature.

Keywords: Moving interfaces, free surfaces, two-fluid flows, computational fluid mechanics, verification and validation.

\section{INTRODUCCIÓN}

El estudio de la evolución temporal de superficies libres e interfaces móviles es relevante en diversos problemas de la ingeniería actual. Pueden mencionarse, por ejemplo, el transporte de líquidos confinados al interior de un tanque, el flujo en un canal, llenado de depósitos, el derrame de un fluido producto de la ruptura de su contenedor o de una compuerta que lo contiene, así como procesos de refinamiento de metales (flujos en cucharas, convertidores).

En este trabajo se presenta el estudio del derrame de una columna de líquido. Este problema fue originalmente analizado experimental [1 y 2], analítica [1] y numéricamente [2]. Actualmente constituye un modelo físico de análisis para la evaluación de técnicas numéricas que permiten la descripción de superficies libres (como en [1-7], por

\footnotetext{
1 Departamento de Ingeniería Mecánica, Universidad de Santiago de Chile, Av. Bernardo O’Higgins 3363. Santiago - Chile. mcruchag@lauca. usach.cl
} 
ejemplo). Más recientemente, se realizó un estudio experimental del problema utilizando dos fluidos de muy diferente viscosidad (agua y shampoo; este último es aproximadamente $10^{4}$ veces más viscoso que el primero [8]) para columnas de líquido con relaciones de aspecto (alto/ancho) igual a 2. Además, en dicho trabajo se realizó el análisis numérico para ambos líquidos y la mencionada relación de aspecto utilizando una técnica de malla fija basada en el método de elementos finitos (MEF) donde para la descripción de la evolución de la superficie interfaz se utilizó una ecuación puramente convectiva de una función marcadora [7].

La modelación que se presenta en este trabajo también utiliza un método de malla fija definido en el marco del método de volúmenes finitos (MVF). Para el seguimiento de la superficie libre, el modelo utiliza la técnica VOF ("Volume Of Fluid" [3 y 9]) que resuelve la ecuación de convección pura que define la posición de la interfaz a través de las fracciones de volumen de cada fluido.

El propósito del presente trabajo es estudiar el comportamiento del MVF-VOF de [9] utilizando diferentes discretizaciones espaciales. La posición de la superficie interfaz se contrasta con los resultados de las simulaciones realizadas en [8] con un modelo descrito en el contexto del MEF [7]. También se incorpora en el análisis la modelación de la turbulencia para evaluar su efecto en la predicción de la posición de la superficie libre. Además de la verificación numérica de los resultados, se propone su validación experimental con las mediciones reportadas en [8].

Los modelos utilizados en el análisis del problema propuesto se describen brevemente en las secciones siguientes. Sus correspondientes discretizaciones numéricas se pueden consultar en [9].

\section{ECUACIONES FUNDAMENTALES Y MODELOS APLICADOS}

En este apartado se presenta el modelo matemático adoptado para el análisis del problema estudiado. Se asume que la dinámica de fluidos es descrita por la ecuación de continuidad (1) y la ecuación de cantidad de movimiento (2) dadas por [10]:

$$
\begin{gathered}
\nabla \cdot \vec{v}=0 \\
\rho\left(\frac{\partial \vec{v}}{\partial t}+\vec{v} \cdot \nabla \vec{v}\right)=-\nabla p+\mu \nabla^{2} \vec{v}+\rho \vec{g}
\end{gathered}
$$

donde se considera al fluido new toniano e incompresible. En estas ecuaciones, $\rho$ es la densidad, $\mu$ es la viscosidad, $\vec{v}$ es el vector velocidad, $p$ es la presión y $\vec{g}$ es el vector gravedad. Los símbolos $\partial / \partial t$ y $\nabla$ representan la derivada parcial temporal y el gradiente espacial, respectivamente.

\section{MODELO VOF}

Para la descripción de la superficie libre se utiliza un modelo donde el volumen ocupado por un material no puede ser ocupado por el otro, dando lugar al concepto básico de fracciones de volumen [3,9]. Éstas representan funciones continuas en el espacio y tiempo, donde la suma total de dichas fracciones, en un punto espacial dado para todo tiempo, debe ser igual a uno. Para actualizar la interfaz se utiliza la ecuación de convección pura de conservación de las especies $\left(\alpha_{q}\right.$, con $q=1,2$ que representan a las fracciones de líquido y aire):

$$
\frac{\partial \alpha_{q}}{\partial t}+\vec{v} \cdot \nabla \alpha_{q}=0
$$

Es importante destacar que la ecuación de conservación de la fracción de volumen se resuelve para una de las fases, ya que la otra se estima a partir de la expresión siguiente:

$$
\sum_{q=1}^{2} \alpha_{q}=1
$$

Para la solución de las ecuaciones (1) y (2) en la discretización de dominio fijo se ponderan las propiedades del fluido de acuerdo a la distribución de la fracción de volumen de cada medio (líquido y aire) dada por (3) y (4).

\section{MODELO DE TURBULENCIA K- $\varepsilon$ STANDARD}

Para incluir efectos de disipación turbulenta se utiliza el modelo k- $\varepsilon$ standard [9 y 10], siendo k la energía cinética turbulenta y $\varepsilon$ la tasa de disipación. Los parámetros $\mathrm{k}$ y $\varepsilon$ del modelo, se obtienen de la ecuación (5) y (6), respectivamente:

$$
\begin{aligned}
\frac{\partial}{\partial t}(\rho k)+\frac{\partial}{\partial x_{i}}\left(\rho k u_{i}\right)= & \frac{\partial}{\partial x_{j}}\left[\left(\mu+\frac{\mu_{t}}{\sigma_{K}}\right) \frac{\partial k}{\partial x_{j}}\right]+ \\
& +G_{k}+G_{b}-\rho \varepsilon-Y_{M}+S_{K}
\end{aligned}
$$




$$
\begin{aligned}
\frac{\partial}{\partial t}(\rho \varepsilon)+\frac{\partial}{\partial x_{i}}\left(\rho \varepsilon u_{i}\right)=\frac{\partial}{\partial x_{j}} & {\left[\left(\mu+\frac{\mu_{t}}{\sigma_{\varepsilon}}\right) \frac{\partial \varepsilon}{\partial x_{j}}\right]+} \\
+ & C_{1 \varepsilon} \frac{\varepsilon}{k}\left(G_{k}+C_{3 \varepsilon} G_{b}\right)-C_{2 \varepsilon} \rho \frac{\varepsilon^{2}}{k}+S_{\varepsilon}
\end{aligned}
$$

donde $\mathrm{G}_{\mathrm{k}}$ representa la generación de energía cinética de turbulencia debido al gradiente medio de la velocidad, $\mathrm{G}_{\mathrm{b}}$ es la generación de energía cinética de turbulencia debido a la flotación, $\mathrm{Y}_{\mathrm{M}}$ representa la contribución de la dilatación fluctuante en la turbulencia comprimible a la proporción de dispersión global, $\sigma_{\mathrm{k}}$ representa al número de Prandtl turbulento para $\mathrm{k}, \sigma_{\varepsilon}$ representa al número de Prandtl turbulento para $\varepsilon, \mathrm{S}_{\mathrm{k}}$ es un término fuente para $\varepsilon, \mathrm{S}_{\varepsilon}$ es un término fuente para $\mathrm{k} y$ el conjunto de parámetros $\mathrm{C}_{1 \varepsilon}$, $\mathrm{C}_{2 \varepsilon}, \mathrm{y}_{3 \varepsilon}$ son constantes a determinar experimentalmente. De acuerdo a la literatura [9], en este trabajo se adoptan los siguientes valores para los mencionados parámetros cuando se utiliza este modelo turbulento en el análisis:

$$
\begin{gathered}
C_{1 \varepsilon}=1.44, \quad C_{2 \varepsilon}=1.92, \quad C_{\mu}=0.091 \\
\sigma_{k}=1.0, \quad \sigma_{\varepsilon}=1.3
\end{gathered}
$$

\section{PRESENTACIÓN DEL PROBLEMA: DESCRIPCIÓN FÍSICA}

El problema estudiado se presenta esquemáticamente en la figura 1 y corresponde al colapso de una columna de fluido producto de la apertura de la compuerta que lo sostiene en el interior de un recipiente. La columna de fluido se derrama por efectos gravitatorios. Los fluidos de trabajo fueron: un fluido viscoso, correspondiente a un shampoo, y agua. El fluido, confinado al recipiente de dimensiones 4L x 4L como muestra la figura 1, está inicialmente en reposo y es contenido entre la compuerta y la pared lateral izquierda del recipiente (zona A) ocupando hasta la altura $\mathrm{H}=2 \mathrm{~L}$, con $\mathrm{L}=0.114 \mathrm{~m}$. Repentinamente la compuerta es retirada y el fluido cae por efecto de la gravedad llenando gradualmente a la Zona B.

Las propiedades físicas adoptadas en la simulación se presentan en la tabla 1. Además, se realizan análisis con modelos de flujo laminar y turbulento.

La simulación del problema físico descrito se realiza con el método de volúmenes finitos utilizando el modelo de fracción de volumen brevemente presentado en el apartado anterior.

En el presente análisis se utilizaron cuatro mallas estructuradas uniformes de 20 x 20, 40 x 40, 60 x 60 y
$80 \times 80$ nodos. Las unidades de trabajo se adoptan todas en el Sistema Internacional (S.I.).

Los casos fueron estudiados hasta los $1.4 \mathrm{~s}$ desde la apertura de la compuerta, donde el paso de tiempo para el análisis numérico fue de $0.001 \mathrm{~s}$.

La aceleración de gravedad, utilizada en todos los casos, fue de $9.81 \mathrm{~m} / \mathrm{s}^{2}$.

\section{ESTUDIO COMPARATIVO DEL COLAPSO DE UNA COLUMNA DE FLUIDO VISCOSO: PRESENTACIÓN, DISCUSIÓN Y VALIDACIÓN DE LOS RESULTADOS}

En este apartado se presenta el análisis numérico del colapso de una columna de fluido viscoso cuyas propiedades, que corresponden a un "shampoo", están reportadas en la tabla 1. Para este caso, el flujo es considerado laminar.

En la figura 2 se muestran las gráficas de la evolución temporal de los puntos $\mathrm{P}_{\mathrm{A}}, \mathrm{P}_{\mathrm{B}} \mathrm{y} \mathrm{P}_{\mathrm{C}}$, que corresponden a la posición de la interfaz sobre la pared izquierda, fondo y pared derecha del recipiente, respectivamente (según figura 1), cuando se utilizan mallas de 20 x 20 y 80 x 80 .

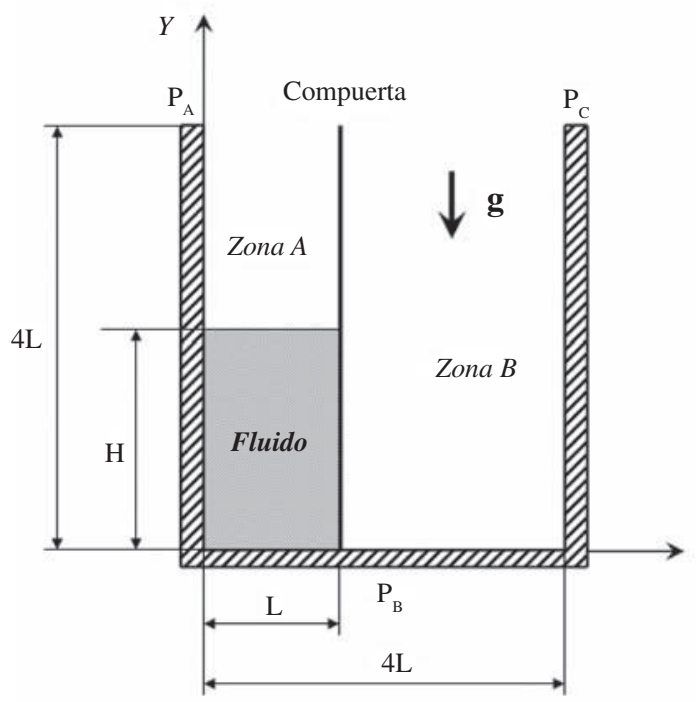

Donde L: Largo característico.

$\mathrm{P}_{\mathrm{A}}:$ Pared lateral izquierda.

$\mathrm{P}_{\mathrm{B}}$ : Pared inferior.

$\mathrm{P}_{\mathrm{C}}$ : Pared lateral derecha.

$\mathrm{H}$ : Altura inicial de fluido de trabajo

(fluido viscoso o agua).

g: Aceleración de gravedad.

Figura 1. Situación física del problema, dimensiones y condiciones iniciales. 
Tabla 1. Propiedades físicas de los fluidos utilizados.

\begin{tabular}{|c|c|c|}
\hline Fluido & $\begin{array}{c}\rho \\
\mathbf{k g} / \mathbf{m}^{3}\end{array}$ & $\begin{array}{c}\mu \\
\mathbf{k g} / \mathbf{m s}\end{array}$ \\
\hline $\begin{array}{c}\text { Fluido viscoso } \\
\text { (shampoo) }\end{array}$ & 1042 & 8 \\
\hline Agua & 1000 & $1 \times 10^{-3}$ \\
\hline Aire & 1 & $1 \times 10^{-6}$ \\
\hline
\end{tabular}

En la figura 3 se presentan los resultados obtenidos con una malla de 20 × 20 para el avance del frente de fluido viscoso en algunos instantes del análisis. Las figura 4 a figura 6 muestran los resultados del mismo problema obtenidos con mallas de 40 x 40, 60 × 60 y 80 x 80 nodos, respectivamente.
En las figuras 2 a 6 se puede apreciar que el fluido viscoso choca con la pared derecha del recipiente antes de $\mathrm{t}=0.30 \mathrm{~s}$, alcanzando la altura máxima en aproximadamente $\mathrm{t}=0.50 \mathrm{~s}$. También se observa que en $\mathrm{t}=1.20 \mathrm{~s}$ el fluido viscoso alcanza la altura máxima sobre la pared izquierda después del choque con la misma. Estos resultados son muy similares entre sí mostrando una cierta independencia de las predicciones obtenidas con distintas discretizaciones espaciales. Sin embargo, las alturas máximas alcanzadas sobre la pared derecha $\left(\mathrm{P}_{\mathrm{c}}\right)$ difieren más significativamente con la discretización. La descripción de la evolución de la interfaz obtenida con VOF es muy semejante a la reportada en [8] utilizando una técnica de seguimiento definida en el marco del MEF.

Evolución de la posición en $\mathrm{P}_{\mathrm{A}}$
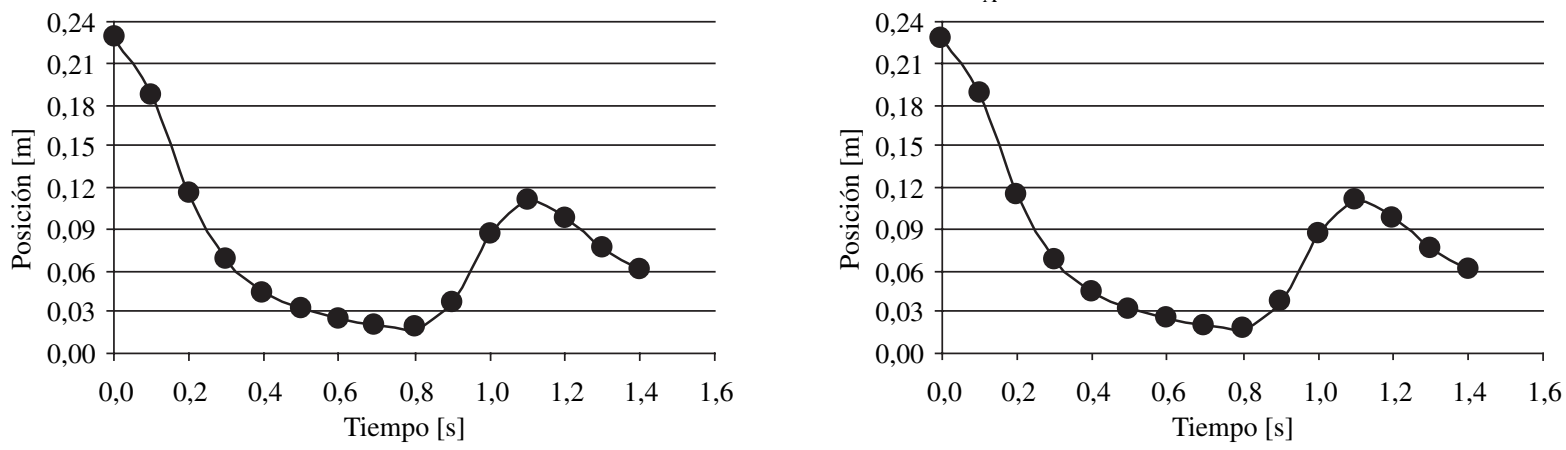

Evolución de la posición en $\mathrm{P}_{\mathrm{B}}$
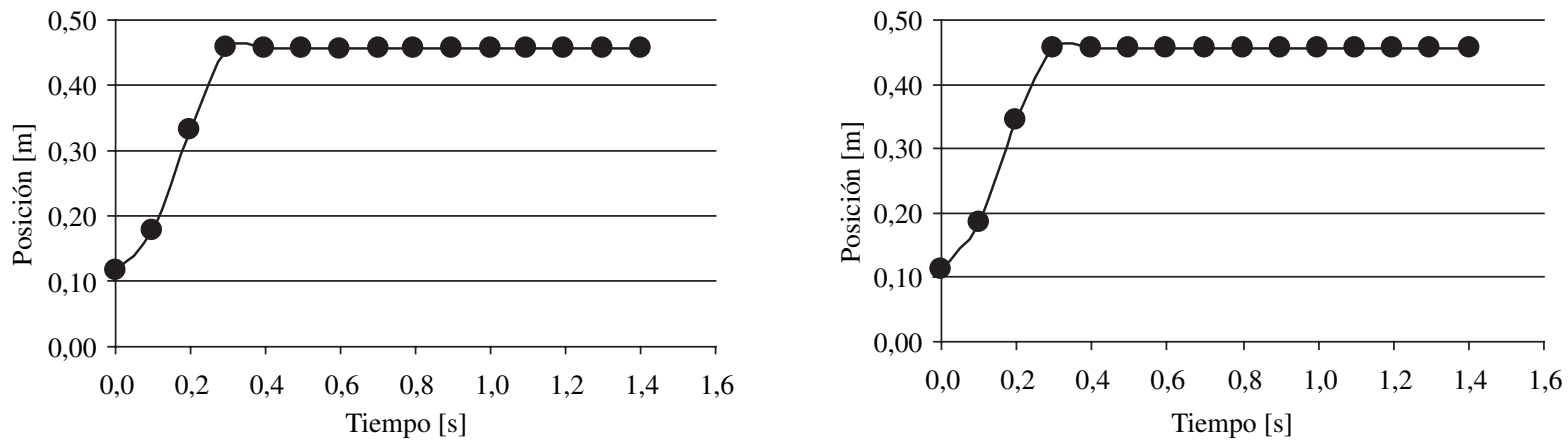

Evolución de la posición en $\mathrm{P}_{\mathrm{C}}$

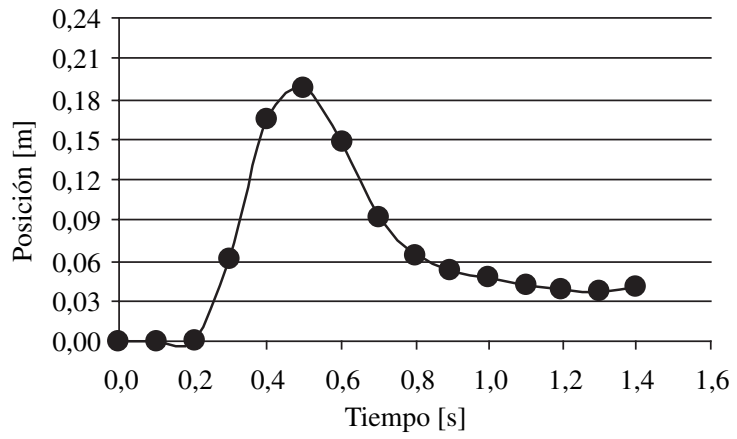

malla $20 \times 20$

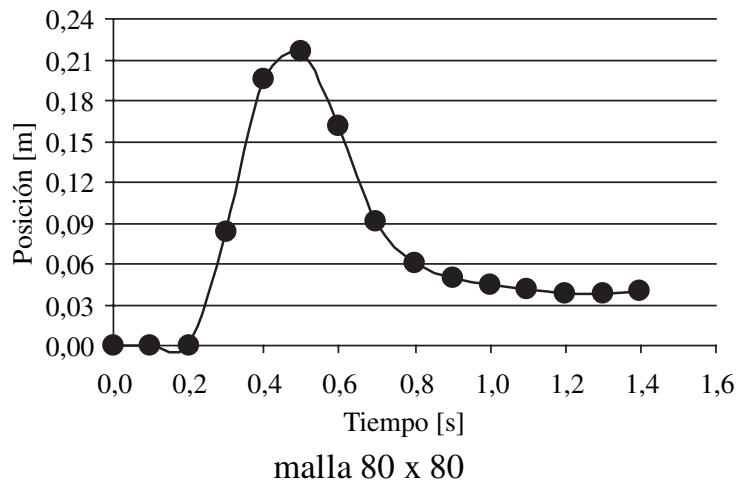

malla $80 \times 80$

Figura 2. Evolución de la posición de la interfaz "shampoo" - aire. 

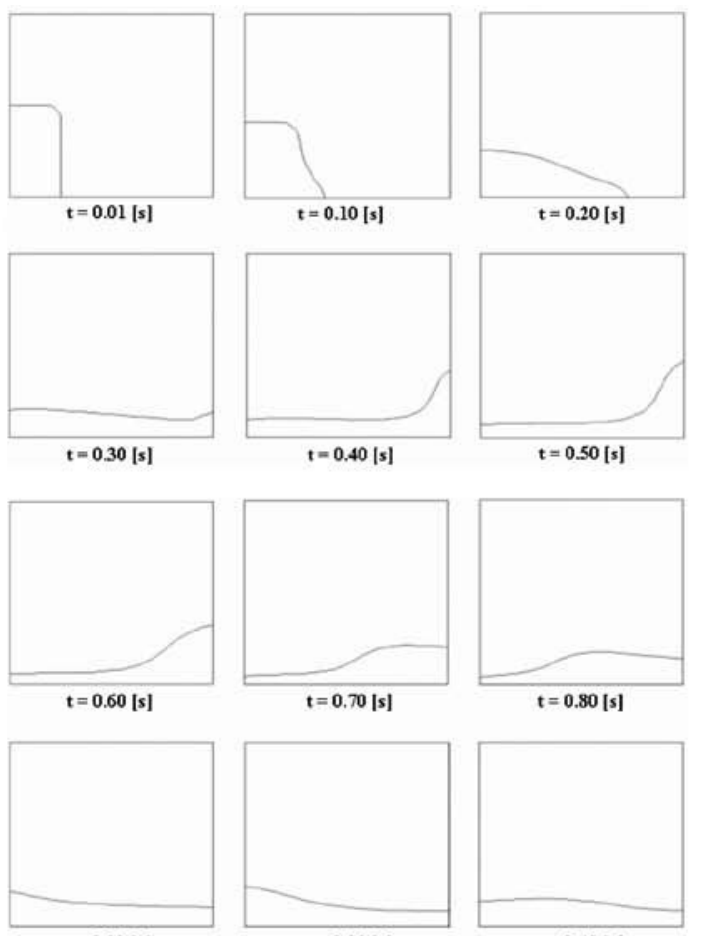

$t=1.20[s]$
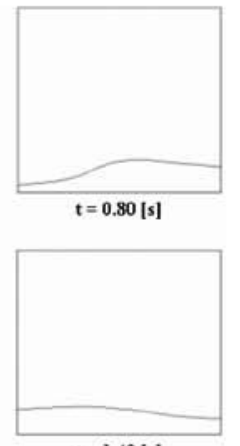

$t=1.40[s]$

Figura 3. Posición de la interfaz del "shampoo" en distintos instantes del análisis con una malla de $20 \times 20$.
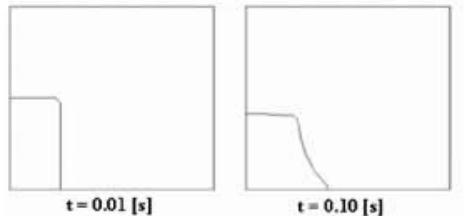

$t=0.10[s]$
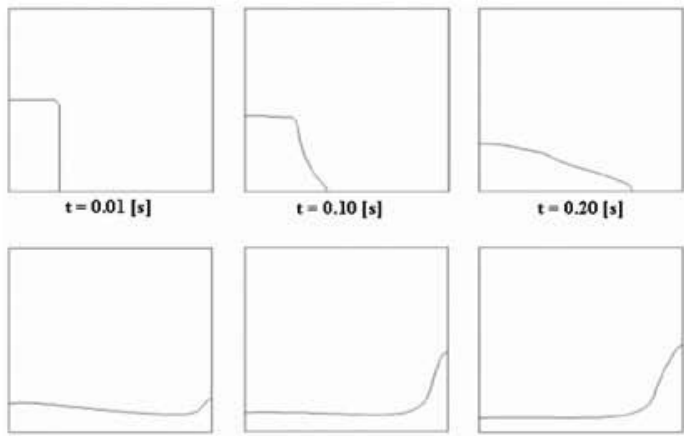

$\mathbf{t}=\mathbf{0 . 3 0}[\mathbf{s}]$
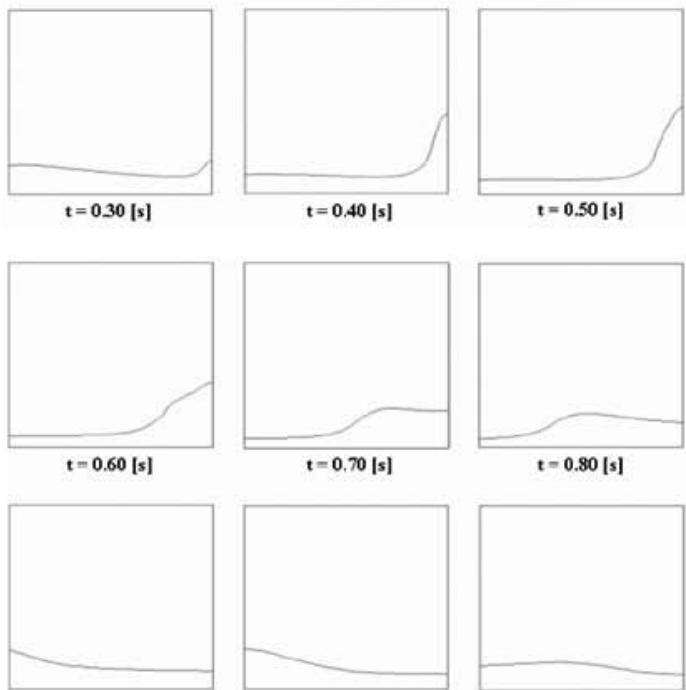

$t=1.00[s]$
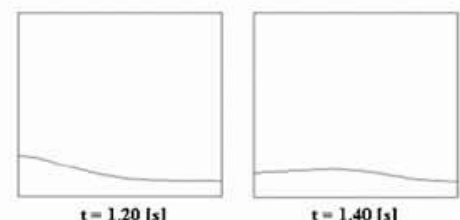

$t=1.40[s]$

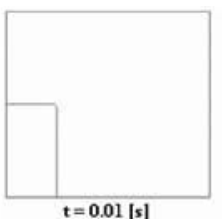

$t=0.01[s]$

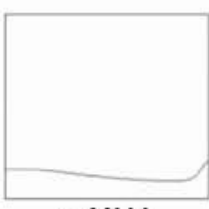

$\mathbf{t}=\mathbf{0 . 3 0}[\mathbf{s}]$

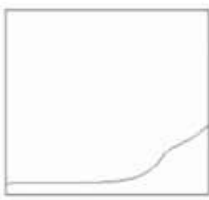

$t=0.60[s]$

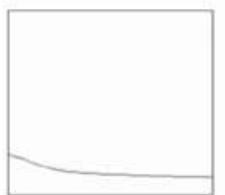

$t=1.00[s]$

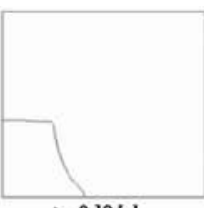

$t=0.10[s]$

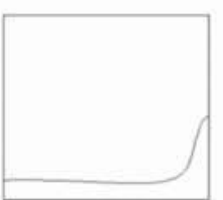

$t=0.40[s]$

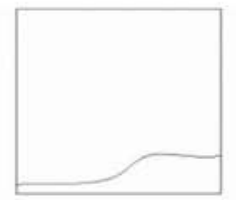

$t=0.70[s]$

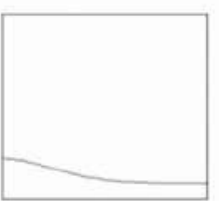

$t=1.20[s]$
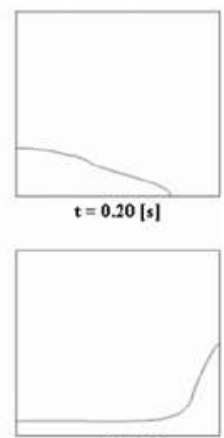

$t=0.50[s]$

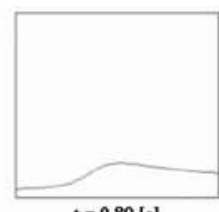

$t=0.80[s]$

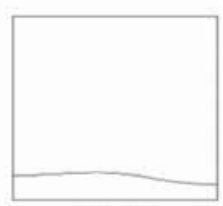

$t=1.40[s]$

Figura 5. Posición de la interfaz del "shampoo" en distintos instantes del análisis con una malla de $60 \times 60$.
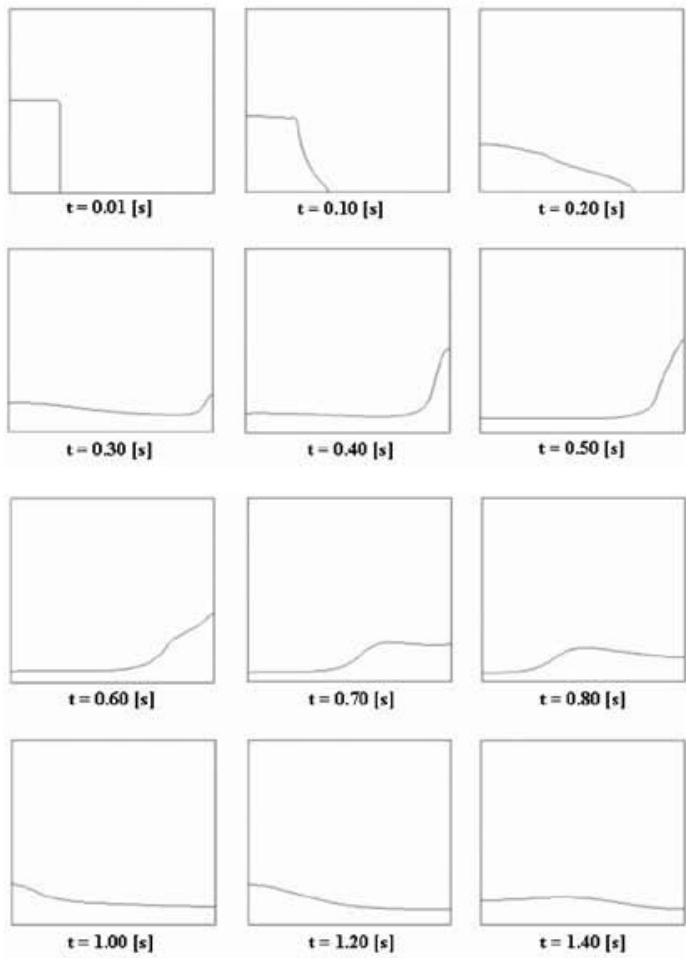

Figura 6. Posición de la interfaz del "shampoo" en distintos instantes del análisis con una malla de $80 \times 80$. 
Evolución temporal del frente de fluido viscoso para $\mathbf{H}=\mathbf{2 L}$ hasta que choca con la pared PC

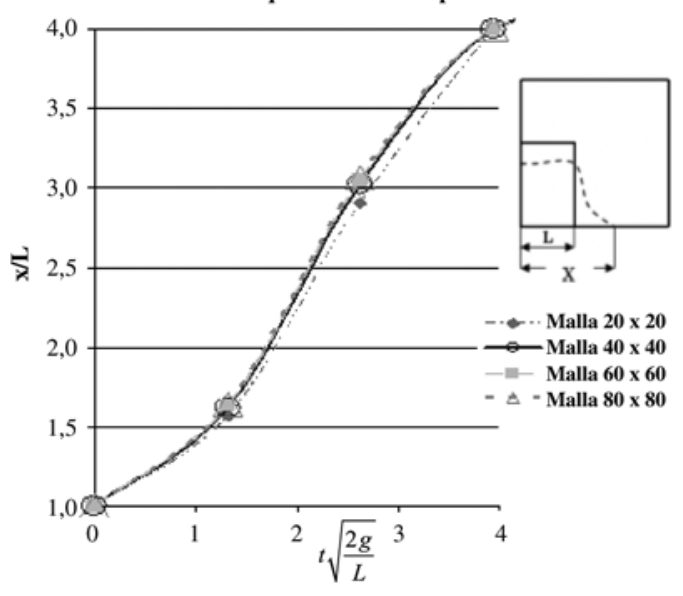

Figura 7. Comparación adimensional de la evolución del frente del "shampoo" sobre la pared inferior $\mathrm{P}_{\mathrm{B}}$.

En la figura 7 se muestra una gráfica comparativa adimensional (siendo la longitud adimensional $\mathrm{x} / \mathrm{L}$ y el tiempo adimensional $t(2 \mathrm{~g} / \mathrm{L})^{1 / 2}$ ) para todas las mallas utilizadas, donde se observa el avance del frente de fluido viscoso en la pared inferior $\left(\mathrm{P}_{\mathrm{B}}\right)$ del recipiente hasta que choca con la pared de la derecha. Puede apreciarse que los resultados son prácticamente coincidentes confirmando cierta independencia con la discretización espacial para la descripción del movimiento hasta el tiempo de choque con la pared izquierda.

En la tabla 2 se presenta la comparación entre los resultados obtenidos en el presente trabajo y los valores reportados en [8] para: la altura máxima alcanzada por el frente de material $\left(\mathrm{h}_{\mathrm{MAX}}\right)$ sobre la pared derecha $\mathrm{P}_{\mathrm{C}}$, el tiempo en que se alcanza esa altura máxima $\left(\mathrm{t}_{\mathrm{MAX}}\right)$, la altura máxima alcanzada después del choque sobre la pared izquierda $\mathrm{P}_{\mathrm{A}}\left(\mathrm{h}_{\mathrm{R}}\right)$ y el tiempo en que la ola generada hacia la pared izquierda alcanza dicha pared $\left(t_{R}\right)$. Dicha tabla muestra que los resultados obtenidos en el presente análisis son similares a los reportados en [8]. Respecto a las mediciones experimentales (cuyo error es de $\pm 0.125 \mathrm{~s}$ en el tiempo y $\pm 0.01 \mathrm{~m}$ en el espacio) se observa que los resultados son bastante cercanos a los de la simulación numérica, a excepción de la malla de 20 x 20 nodos que no captura en forma satisfactoria el fenómeno, en particular, en la descripción de la altura máxima después del primer choque (sobre la pared derecha).

La figura 8 muestra la posición del frente material para dos instantes, $\mathrm{t}=0.70 \mathrm{~s}$ y $\mathrm{t}=1.20 \mathrm{~s}$, obtenidas en el presente trabajo junto a las interfaces calculadas en [8]. La comparación entre los resultados numéricos y las posiciones registradas experimentalmente (también incluidas en la misma figura) es satisfactoria.

Tabla 2. Comparación de tiempos y alturas alcanzadas por el frente del "shampoo".

\begin{tabular}{|c|c|c|c|c|}
\hline Caso & $\begin{array}{c}\mathbf{h}_{\text {MAX }} \\
{[\mathbf{m}]}\end{array}$ & $\begin{array}{c}\mathbf{t}_{\mathbf{M A X}} \\
{[\mathbf{s}]}\end{array}$ & $\begin{array}{c}\mathbf{h}_{\mathbf{R}} \\
{[\mathbf{m}]}\end{array}$ & $\begin{array}{c}\mathbf{t}_{\mathbf{R}} \\
{[\mathbf{s}]}\end{array}$ \\
\hline $20 \times 20[\mathbf{8}]$ & 0.158 & 0.42 & 0.110 & 1.00 \\
\hline $20 \times 20[\mathbf{P T}]$ & 0.188 & 0.50 & 0.110 & 1.10 \\
\hline $40 \times 40[\mathbf{8}]$ & 0.230 & 0.48 & 0.114 & 1.00 \\
\hline $40 \times 40[\mathbf{P T}]$ & 0.215 & 0.50 & 0.118 & 1.10 \\
\hline $60 \times 60[\mathbf{8}]$ & 0.236 & 0.48 & 0.114 & 1.00 \\
\hline $60 \times 60[\mathbf{P T}]$ & 0.224 & 0.50 & 0.120 & 1.10 \\
\hline $80 \times 80[\mathbf{8}]$ & 0.297 & 0.48 & 0.114 & 1.00 \\
\hline $80 \times 80[\mathbf{P T}]$ & 0.228 & 0.50 & 0.121 & 1.10 \\
\hline Experimental [8] & 0.220 & 0.48 & 0.075 & 1.00 \\
\hline
\end{tabular}

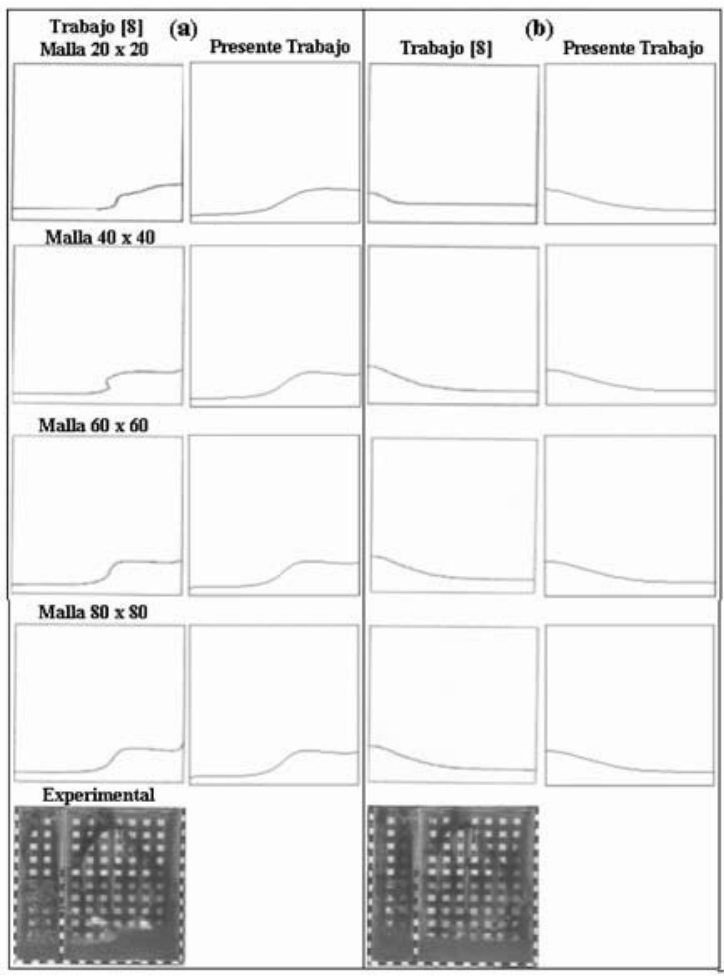

Figura 8. Comparación de la posición del frente del "shampoo" en los tiempos (a) $t=0.7$ y (b) $\mathrm{t}=1.2 \mathrm{~s}$ considerando distintas discretizaciones y métodos de cálculo.

\section{ESTUDIO COMPARATIVO DEL COLAPSO DE UNA COLUMNA DE AGUA: PRESENTACIÓN, DISCUSIÓN Y VALIDACIÓN DE LOS RESULTADOS}

En este apartado se presentan los resultados del colapso de una columna de agua (cuyas propiedades se presentan en la tabla 1) para una relación de aspecto de 2 con iguales 
dimensiones que las reportadas en el apartado anterior. Se realizan diversas simulaciones donde, además de las diferentes discretizaciones espaciales ya presentadas, se utilizan dos modelos de flujo: uno laminar y otro turbulento (de acuerdo a la descripción dada previamente en el correspondiente apartado) con la finalidad de establecer la influencia de dichos modelos en la descripción del frente material.

En las figuras 9 y 10 se muestran las gráficas de la evolución temporal de la superficie interfaz en la pared lateral izquierda $\mathrm{P}_{\mathrm{A}}$, en la base $\mathrm{P}_{\mathrm{B}} \mathrm{y}$ en la pared lateral derecha $\mathrm{P}_{\mathrm{C}}$ (según figura 1) obtenidas con las diversas mallas utilizadas $y$ usando un modelo laminar. En dichas figuras se aprecian diferencias en la predicción de las alturas máximas alcanzadas sobre la pared derecha y la izquierda cuando se modifica la discretización espacial. Sin embargo, las diferencias no son significativas entre los resultados obtenidos con discretizaciones mayores a $60 \times 60$.

En la figura 11 se muestra el avance del frente de agua a lo largo de la pared inferior $\left(\mathrm{P}_{\mathrm{B}}\right)$ hasta que choca con la pared de la derecha $\left(\mathrm{P}_{\mathrm{C}}\right)$. En dicha figura se presentan los resultados en forma adimensional y se observa que la evolución hasta ese instante es prácticamente independiente de la discretización espacial.

Evolución de la posición en $\mathrm{P}_{\mathrm{A}}$
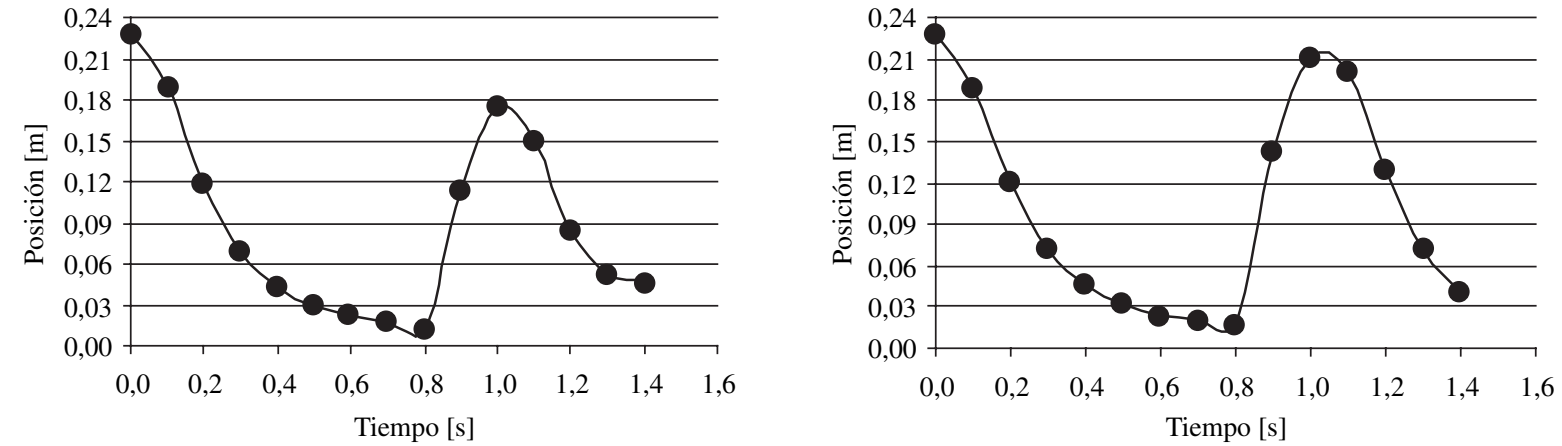

Evolución de la posición en $\mathrm{P}_{\mathrm{B}}$
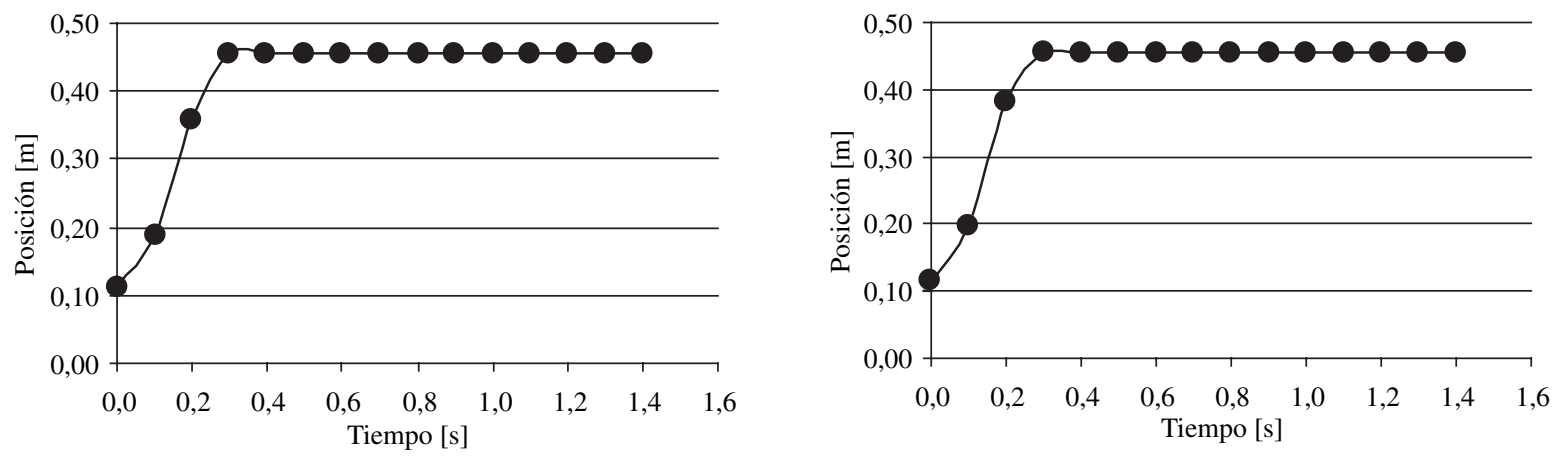

Evolución de la posición en $\mathrm{P}_{\mathrm{C}}$

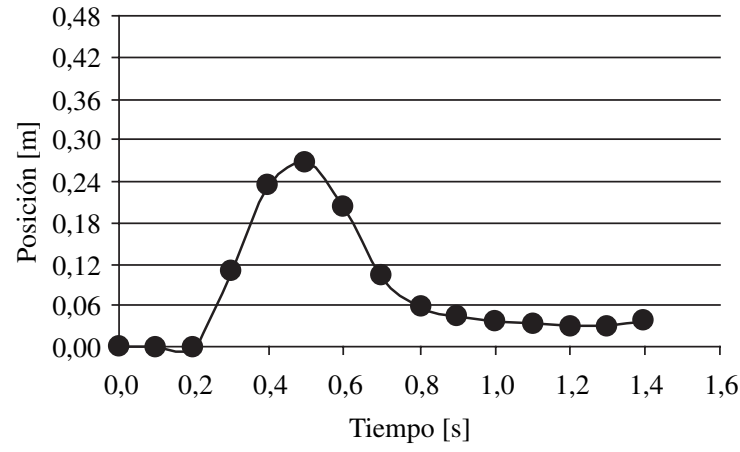

malla $20 \times 20$

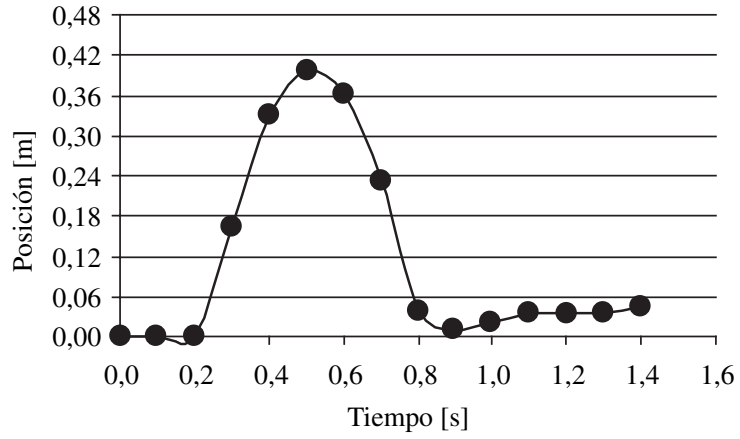

malla $40 \times 40$

Figura 9. Evolución de la posición de la interfaz agua-aire (modelo laminar). 
Evolución de la posición en $\mathrm{P}_{\mathrm{A}}$
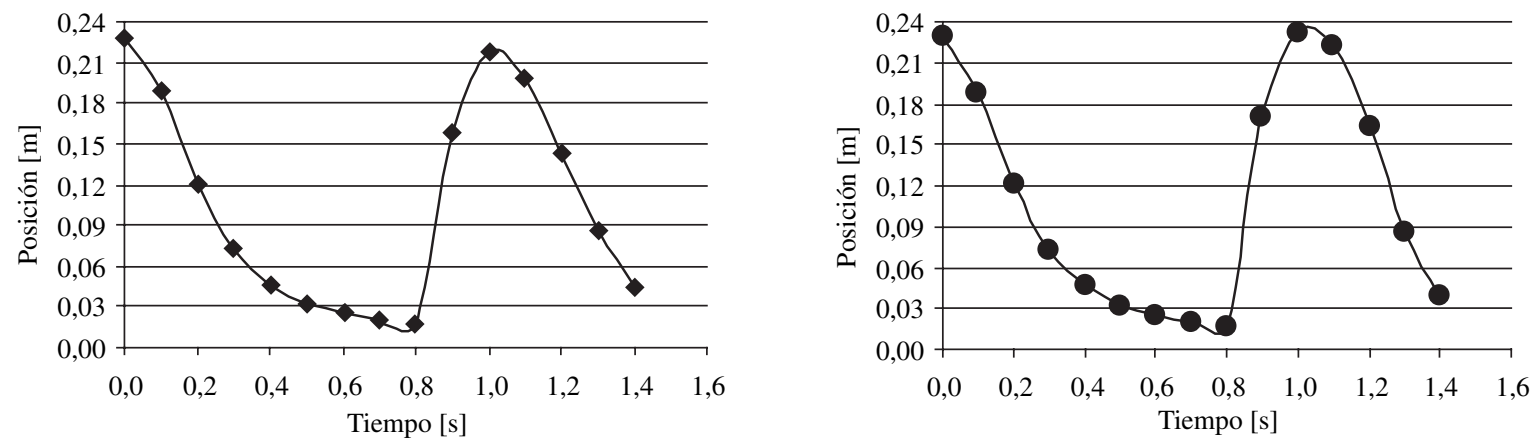

Evolución de la posición en $\mathrm{P}_{\mathrm{B}}$
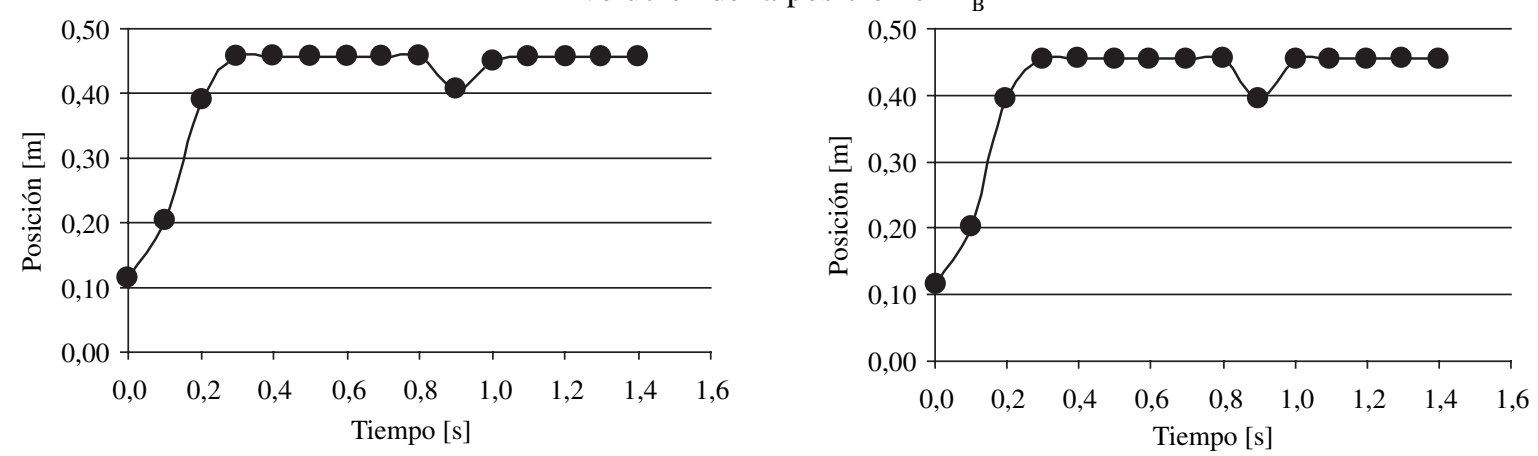

Evolución de la posición en $\mathrm{P}_{\mathrm{C}}$

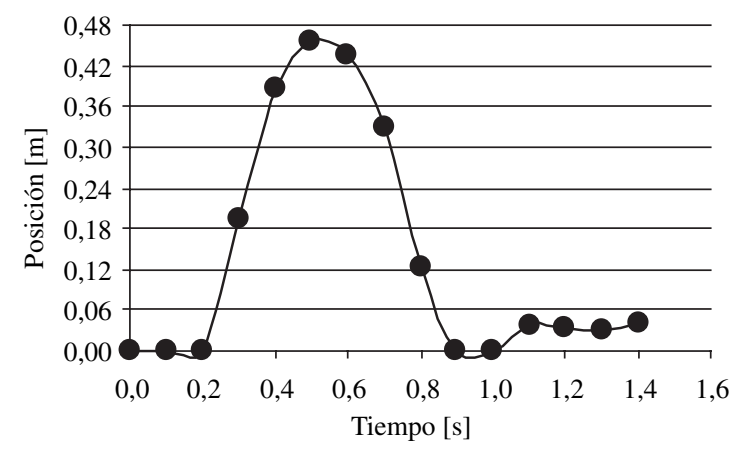

malla $60 \times 60$

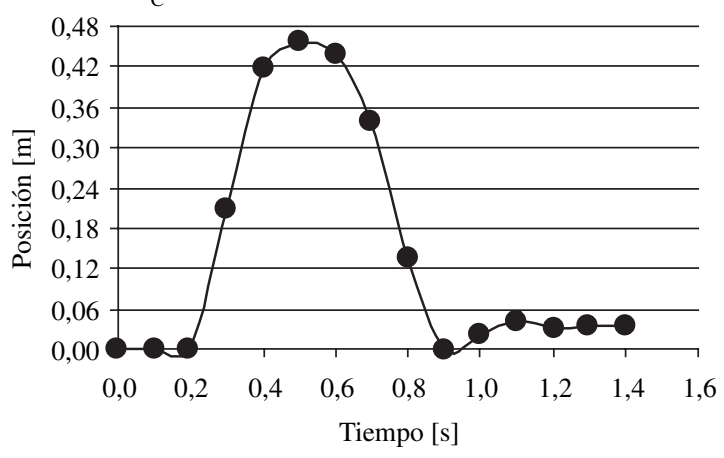

Figura 10. Evolución de la posición de la interfaz agua-aire (modelo laminar).

En la tabla 3 se comparan algunos parámetros importantes obtenidos en el presente trabajo utilizando un modelo laminar y para las diferentes discretizaciones analizadas. Se confirma que si bien hay coincidencia en los instantes para los cuales se registran las alturas máximas alcanzadas en al pared derecha $\left({ }_{\text {MAX }}\right)$ y en la izquierda $\left({ }_{R}\right)$, las predicciones de las magnitudes de dichas alturas máximas se ven afectadas por la densidad de malla (creciendo la altura para discretizaciones más densas).

En las figuras 12 a 15 se presenta la posición de la superficie libre en distintos instantes del análisis obtenidas con un modelo laminar y para las diferentes discretizaciones espaciales utilizadas. Se puede apreciar que el agua luego de chocar con $\mathrm{P}_{\mathrm{C}}$, aproximadamente a los $0.30 \mathrm{~s}$, comienza a subir por esta pared superando incluso, en los análisis con mayor densidad de malla, el límite de la pared del contenedor y continuando su movimiento ascendente en el tiempo. Este comportamiento no es observado en la experiencia. Posteriormente se genera una propagación ondulante del frente y aparecen "burbujas". En la experiencia física se observa la generación del movimiento ondulante predicho. Sin embargo, el flujo se separa pero no forma grandes oquedades en su seno como predice la modelación numérica. Debido a que las simulaciones presentan estas discrepancias, con la observación física se propone realizar un análisis bajo condiciones de flujo turbulento cuyos resultados se presentan a continuación. 


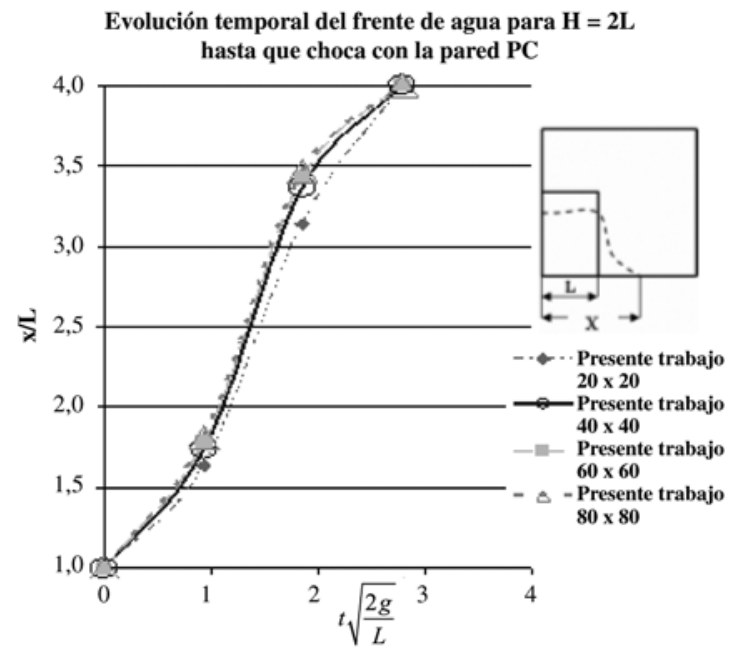

Figura 11. Comparación adimensional de la evolución del frente de agua (modelo laminar) sobre la pared inferior $\mathrm{P}_{\mathrm{B}}$.

En las figuras 16 y 17 se presenta la posición de la interfaz en diferentes tiempos del análisis obtenidos con mallas de 20 × 20 y 80 × 80 , respectivamente, considerando flujo turbulento. Dichas figuras presentan, respecto a las obtenidas bajo la hipótesis de flujo laminar (figura 12 y 15), una interfaz más suave y no muestran "burbujas" en el seno líquido.

En la tabla 4 se comparan los valores obtenidos en este estudio y los reportados en [8] (numéricos y experimentales) para el tiempo en que se producen las alturas máximas alcanzadas en la pared derecha $\left({ }_{\text {MAX }}\right)$ y en la izquierda $\left(_{R}\right)$ y los correspondientes valores de dichas alturas máximas. Si bien los resultados muestran concordancia en la determinación de los tiempos, las alturas máximas predichas en este estudio difieren de las mediciones experimentales y de las obtenidas en la simulación de [8]. Esta diferencia puede deberse a los parámetros utilizados en el modelo turbulento. Para el análisis se adoptaron parámetros comúnmente recomendados. Sin embargo, éstos deberían correlacionarse con observaciones experimentales en la situación estudiada.

Tabla 3. Comparación de tiempos y alturas del frente de agua obtenidas en el presente estudio con un modelo laminar de flujo utilizando diversas discretizaciones espaciales.

\begin{tabular}{|c|c|c|c|c|}
\hline Caso & $\mathbf{H}_{\text {MAX }}[\mathbf{m}]$ & $\mathbf{T}_{\text {MAX }}[\mathbf{s}]$ & $\mathbf{h}_{\mathbf{R}}[\mathbf{m}]$ & $\begin{array}{c}\mathbf{t}_{\mathbf{R}} \\
{[\mathbf{s}]}\end{array}$ \\
\hline $20 \times 20[\mathbf{P T}]$ & 0.27 & 0.5 & 0.17 & 1.0 \\
\hline $40 \times 40[\mathbf{P T}]$ & 0.39 & 0.5 & 0.21 & 1.0 \\
\hline $60 \times 60[\mathbf{P T}]$ & 0.46 & 0.5 & 0.22 & 1.0 \\
\hline $80 \times 80[\mathbf{P T}]$ & 0.46 & 0.5 & 0.23 & 1.0 \\
\hline
\end{tabular}

Tabla 4. Comparación de tiempos y alturas del frente de agua para flujo turbulento.

\begin{tabular}{|c|c|c|c|c|}
\hline Caso & $\begin{array}{c}\mathbf{H}_{\mathbf{M A X}} \\
{[\mathbf{m}]}\end{array}$ & $\begin{array}{c}\mathbf{t}_{\mathbf{M A X}} \\
{[\mathbf{s}]}\end{array}$ & $\begin{array}{c}\mathbf{h}_{\mathbf{R}} \\
{[\mathbf{m}]}\end{array}$ & $\begin{array}{c}\mathbf{t}_{\mathbf{R}} \\
{[\mathbf{s}]}\end{array}$ \\
\hline $20 \times 20[\mathbf{P T}]$ & 0.24 & 0.5 & 0.16 & 1.1 \\
\hline $80 \times 80[\mathbf{P T}]$ & 0.35 & 0.5 & 0.31 & 1.1 \\
\hline $\begin{array}{c}\text { Promedio } \\
\text { Numérico [8] }\end{array}$ & 0.40 & 0.5 & 0.21 & 1.0 \\
\hline $\begin{array}{c}\text { Promedio } \\
\text { Experimental [8] }\end{array}$ & 0.44 & 0.5 & 0.26 & 0.9 \\
\hline
\end{tabular}

\section{CONCLUSIONES}

En este trabajo se presentó un análisis comparativo de dos modelos numéricos diferentes utilizados para la descripción de interfaces móviles y superficies libres. La forma discreta de las ecuaciones diferenciales del modelo matemático con que se describe el problema analizado (ecuaciones de Navier Stokes para flujo incompresible y seguimiento de la interfaz) se escribe en el marco de discretizaciones de malla fija. En particular, en este estudio se utiliza el método de volúmenes finitos junto con el modelo de fracción de volúmenes. Los resultados obtenidos en el análisis del derrame de una columna de líquido se comparan con los de otra técnica definida en el marco del método de elementos finitos y con mediciones experimentales. Se ha analizado, además, el comportamiento de dos fluidos con viscosidades muy distintas evaluando el efecto de dicha propiedad sobre la descripción del comportamiento de la superficie interfaz. Por otro lado, se verificó el efecto de incorporar un modelo turbulento en la descripción del frente material.

Los resultados numéricos para ambos fluidos presentan cierta independencia del tamaño de malla. Los modelos comparados muestran mayor similitud de resultados en el caso del fluido viscoso, los que también presentan un mejor ajuste a los valores experimentales reportados en la literatura. Sin embargo, cuando se considera un fluido con baja viscosidad, los resultados de las simulaciones obtenidos en el presente trabajo para los valores máximos alcanzados por la columna de líquido, tanto para comportamiento laminar como turbulento del flujo, se apartan de los reportados en las referencias mostrando un comportamiento más difusivo.

\section{AGRADECIMIENTOS}

Los autores agradecen al Proyecto Fondecyt 1060141 en el marco del cual se desarrolló el presente estudio. 

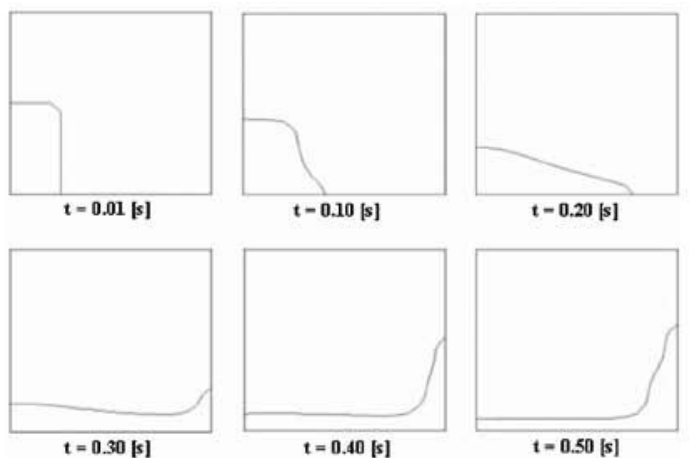

$t=0.40[s]$
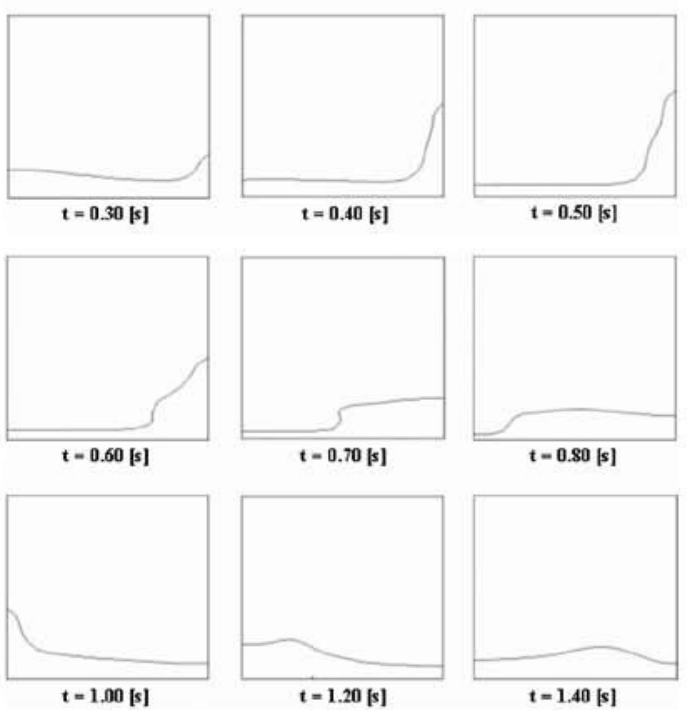

Figura 12.Posición de la interfaz del agua en distintos instantes del análisis con una malla de $20 \times 20$ (modelo laminar).
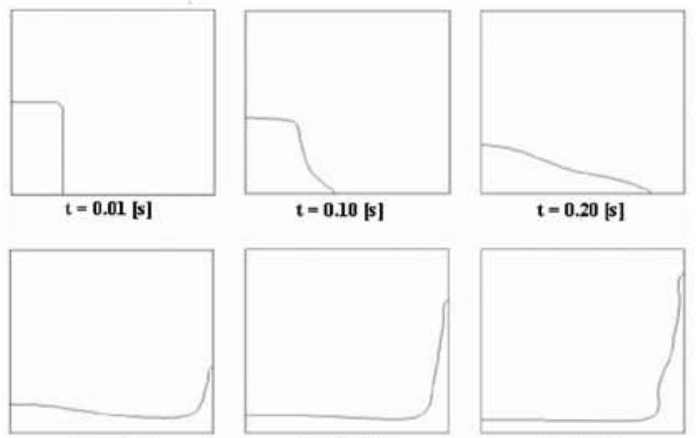

$t=0.40[s]$
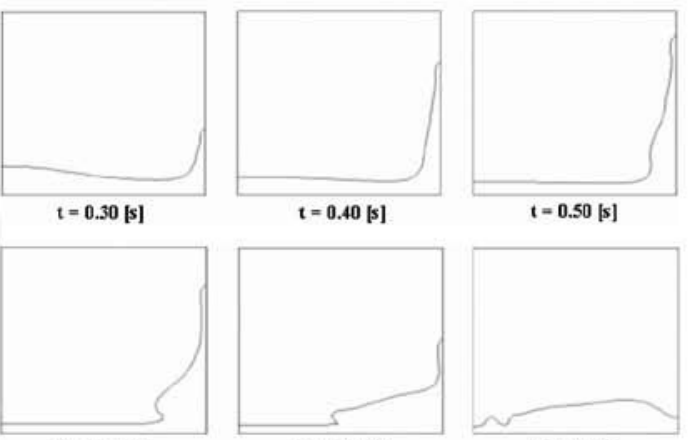

$t=0.60[s]$
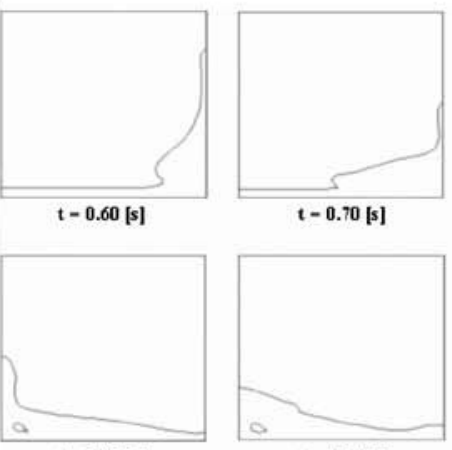

$t=1.00[s]$
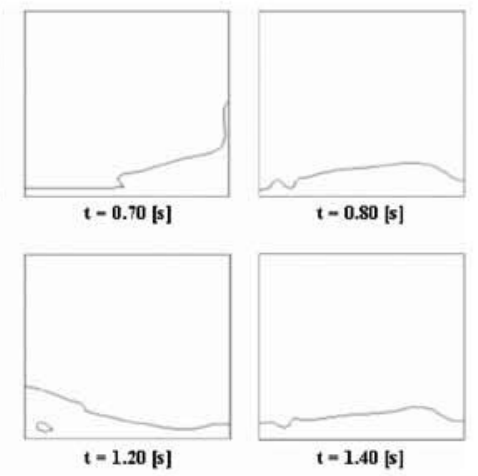

Figura 13. Posición de la interfaz del agua en distintos instantes del análisis con una malla de 40 x 40 (modelo laminar).
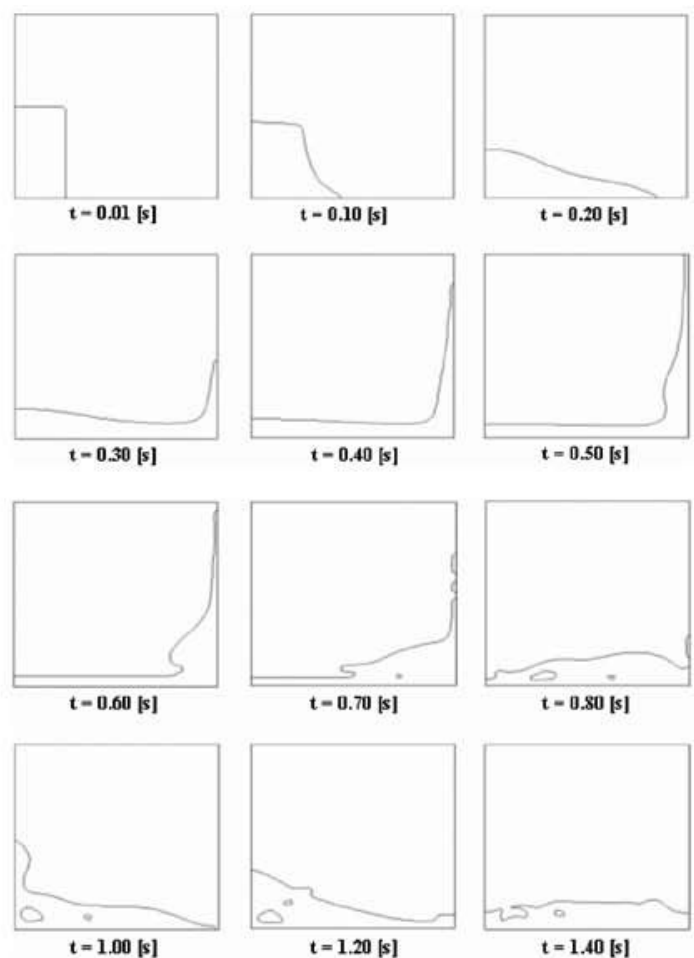

Figura 14. Posición de la interfaz del agua en distintos instantes del análisis con una malla de $60 \times 60$ (modelo laminar).
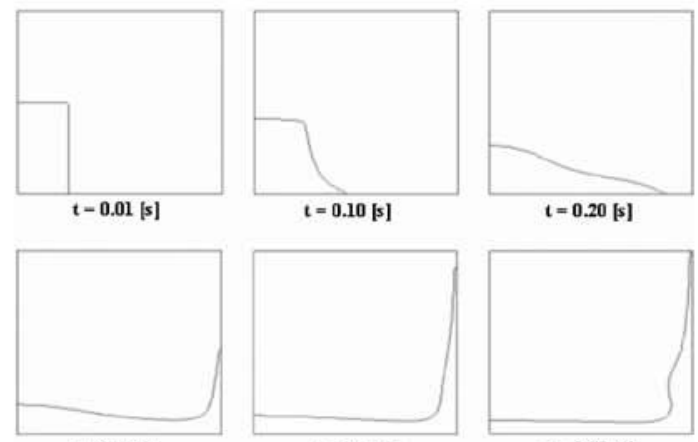

$t=0.30[s]$

$t=0.40[s]$

$\mathrm{t}=0.50[\mathrm{~s}]$
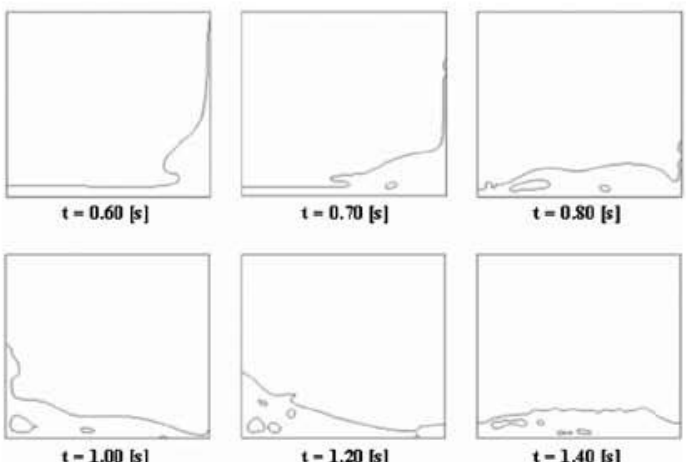

Figura 15. Posición de la interfaz del agua en distintos instantes del análisis con una malla de $80 \times 80$ (modelo laminar). 

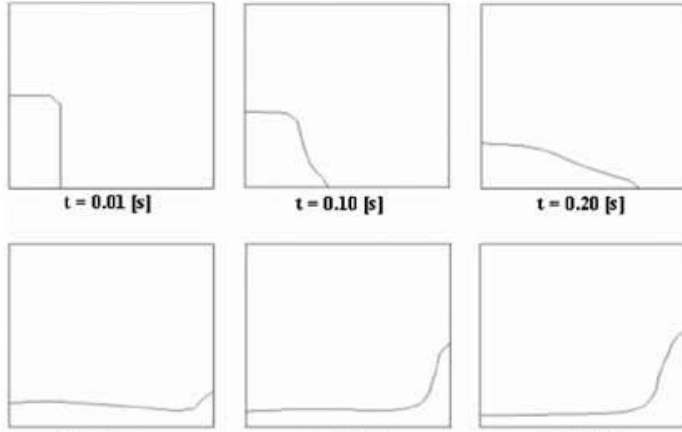

$\mathrm{t}=\mathbf{0 . 3 0}[\mathrm{s}]$

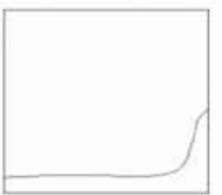

$\mathrm{t}=0.40[\mathrm{~s}]$
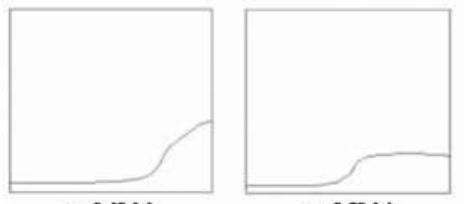

$t=0.70[s]$

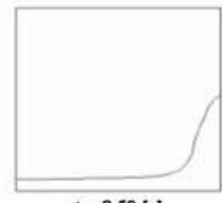

$t=0.50[s]$

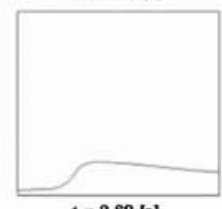

$t=0.60[s]$

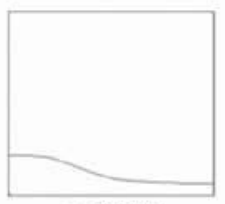

$\mathrm{t}=1.20[\mathrm{~s}]$

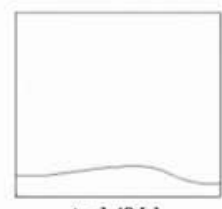

$\mathrm{t}=1.00[s]$

$t=1.40[\mathrm{~s}]$

\section{REFERENCIAS}

[1] J. Martin and W. Moyce. "An experimental study of the collapse of liquid columns on a rigid horizontal plane". Phil. Tran. Royal Society London. Vol. 244, pp. 312-324. 1952.

[2] S. Koshizuka and Y. Oka. "Moving-particle semiimplicit method for fragmentation of incompressible fluid". Nuclear Science and Engineering. Vol. 123, pp. 421-434. 1996.

[3] M.S. Kim and W.I. Lee. "A new VOF-based numerical scheme for the simulation of fluid flow with free surface. Part I: New free surface-tracking algorithm and its verification". International Journal for Numerical Methods in Fluids. Vol. 42, 765-790. 2003.

[4] Wusi Yue, Ch. L. Lin and V.C. Patel, "Numerical simulation of unsteady multidimensional free surface motons by level set method", International Journal for Numerical Methods in Fluids. Vol. 42, pp. 853-884. 2003.

instantes del análisis con una malla de $20 \times 20$ para flujo turbulento.
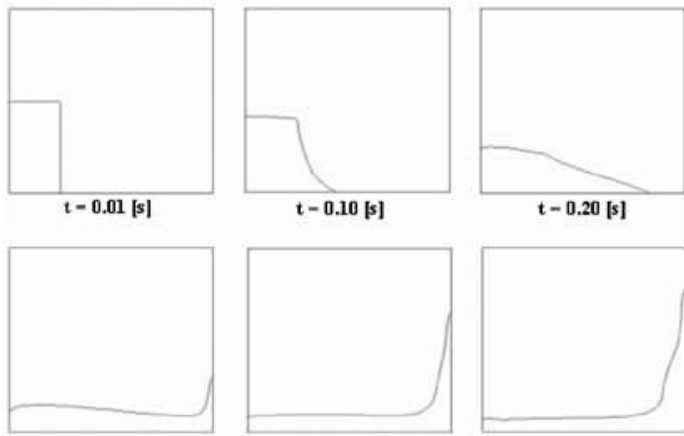

$t=0.30[s]$

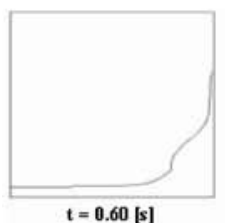

$t=0.60[s]$

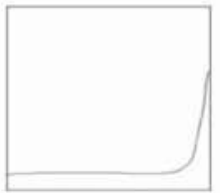

$t=0.40[s]$

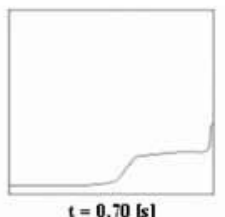

$t=0.70[s]$
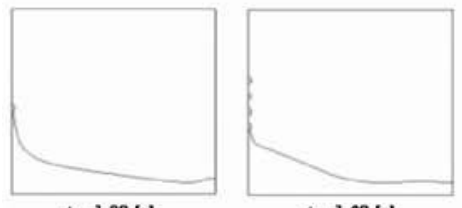

$t-1.20[s]$

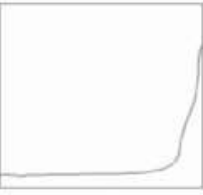

$t-0.50[s]$
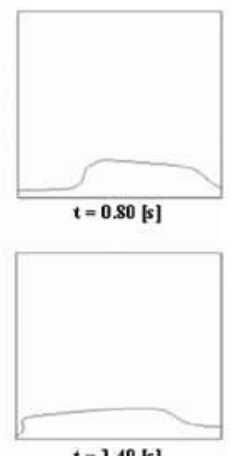

$t=1.40[s]$

H. Kohno and T. Tanahashi. "Numerical analysis of moving interfaces using a level set method coupled with adaptive mesh refinement", International Journal for Numerical Methods in Fluids. Vol. 45, pp. 921-944. 2004.

D. Greaves. "Simulation of interface and free surface flows in a viscous fluid using adapting quadtree grids". International Journal for Numerical Methods in Fluids. Vol. 44, pp. 1093-1117. 2004.

[7] M.A. Cruchaga, D.J. Celentano and T.E. Tezduyar. "Moving-interface computations with the Edgetracked Interface Locator Technique (ETILT)". International Journal for Numerical Methods in Fluids. Vol. 47, pp. 451-469. 2005.

[8] R. Silva. "Modelos Comparativos del Colapso de una Columna de Agua". Tesis para optar al título de Ingeniero Civil Mecánico. Departamento de Ingeniería Mecánica. Universidad de Santiago de Chile. Santiago, Chile. 2004.

FLUENT Inc. FLUENT® User's Guide - Release 5.3. 1999.

[10] F. White. "Mecánica de Fluidos". McGraw - Hill. Cuarta edición. México. 1979. 